\title{
Approximate Moment Dynamics for Chemically Reacting Systems
}

\author{
Abhyudai Singh João Pedro Hespanha \\ Deptment of Electrical and Computer Engineering, \\ University of California at Santa Barbara, \\ Santa Barbara, CA 93101. \\ Email: a2singh@ucsd.edu, hespanha@ece.ucsb.edu
}

\begin{abstract}
In the stochastic formulation of chemical kinetics, the differential equation that describes the time evolution of the lower-order statistical moments for the number of molecules of the different species involved, is generally not closed, in the sense that the right-hand side of this equation depends on higher-order moments. Recent work has proposed a moment closure technique based on derivative-matching, which closes the moment equations by approximating higher-order moments as nonlinear functions of lower-order moments. We here provide a mathematical proof of this moment closure technique, and highlight its performance through comparisons with alternative methods. These comparisons reveal that this moment closure technique based on derivative-matching provides more accurate estimates of the moment dynamics, especially when the population size is small. Finally, we show that the accuracy of the proposed moment closure scheme can be arbitrarily increased by incurring additional computational effort.
\end{abstract}

Index Terms

Stochastic noise, chemical reactions, moment dynamics, moment closure, lognormal distributions, derivativematching.

\section{INTRODUCTION}

The time evolution of a spatially homogeneous mixture of chemically reacting molecules is often modeled using a stochastic formulation, which takes into account the inherent randomness of thermal molecular motion [1]. The stochastic formulation allows the computation of the probability density function for the population counts of the different chemical species involved, which is often done through 
various Monte Carlo techniques [1], [2], [3] and the finite state projection algorithm [4] at a significant computational cost. Since one is often interested in computing only the first and second order statistical moments of the population count, much time and effort can be saved by applying approximate methods (for example, Van Kampen's linear noise approximation [5]) to directly compute these low-order moments, without actually having to solve for the probability density function.

In Section II we show that the differential equations that describe the time evolution of the first $\mathscr{M}$ order statistical moments for the number of molecules of the different species involved, is not closed, in the sense that the right-hand-side of these equations depend on higher-order moments of order $\mathscr{M}+1$. This assertion is based on the assumption that there is at least one bi-molecular reaction (i.e., has two reactants) in the set of chemical reactions considered. For analysis purposes, the moment equations can be closed by approximating higher order statistical moments of order $\mathscr{M}+1$ as nonlinear functions of moments of order up to $\mathscr{M}$. This procedure is commonly referred to in literature as moment closure [6], $[7]$.

A procedure to perform moment closure by matching time derivatives of the exact (not closed) moment equations with that of the approximate (closed) moment equations for some initial time and set of initial conditions has recently been proposed [8], [9]. In Section III we provide a mathematical proof of the main result behind this moment closure technique, which provides explicit analytical formulas to approximate any higher order statistical moment as a nonlinear function of moments of order up to $\mathscr{M}$. Striking features of these nonlinear functions are that they are independent of the reaction parameters (reaction rates and stoichiometry) and moreover the dependence of higher-order moment on lower order ones is consistent with the population being jointly lognormally distributed, in spite of the fact that the moment closure method does not make any a priori assumption on the distribution of the population. Moreover, we show that by increasing $\mathscr{M}$, the closed moment equations provide more accurate approximations of the exact moment dynamics.

Finally, in Section IV, we compare the proposed moment closure scheme to an alternative procedure, where moment closure is performed by setting the third and higher order statistical cumulants of the population count equal to zero [10], [11]. We refer to this latter procedure as the zero-cumulant moment closure. It is shown that when the population size is small, the derivative matching moment closure proposed here outperforms the zero-cumulant moment closure. 
TABLE I

PROPENSITY FUNCTIONS FOR DIFFERENT ELEMENTARY REACTIONS.

\begin{tabular}{lc}
\hline Reaction $R_{i}$ & Probability reaction will occur in interval $(t, t+d t]$ \\
\hline$* \stackrel{c_{i}}{\longrightarrow} X_{j}$ & $c_{i} d t$ \\
$X_{j} \stackrel{c_{i}}{\longrightarrow}$ & $c_{i} x_{j} d t$ \\
$X_{j}+X_{\ell}(\ell \neq j) \stackrel{c_{i}}{\longrightarrow}$ & $c_{i} x_{j} x_{\ell} d t$ \\
$2 X_{j} \stackrel{c_{i}}{\longrightarrow}$ & $\frac{c_{i}}{2} x_{j}\left(x_{j}-1\right) d t$ \\
\hline
\end{tabular}

\section{MOMENT DYNAMICS OF CHEMICALLY REACTING SYSTEMS}

Consider a system of $n$ chemical species $X_{j}, j \in\{1, \ldots, n\}$ involved in $K$ reactions $R_{i}, i \in\{1, \ldots, K\}$ of the form given in the first column of Table I. In the sequel, we denote by $x_{i}(t)$ as the number of molecules of the species $X_{i}$ at time $t$. In the stochastic formulation, each reaction $R_{i}$ is a stochastic event which resets the population count $\mathbf{x}=\left[x_{1}, \ldots, x_{n}\right]^{T}$ based on the map $\mathbf{x} \mapsto \mathbf{x}+\mathbf{a}_{i}$, where the vector $\mathbf{a}_{i}$ is uniquely determined by the stoichiometry of the $i^{\text {th }}$ reaction. The probability that the $i^{\text {th }}$ reaction $R_{i}$ will take place in the next "infinitesimal" time interval $(t, t+d t]$ is given by $h_{i}(\mathbf{x}) d t$, where $h_{i}(\mathbf{x})$ is called the propensity function of reaction $R_{i}$ [1]. Table I shows the form of the propensity functions for different reactions and illustrates that these functions are polynomials in the population count $\mathbf{x}$.

Given a vector $\mathbf{m}=\left(m_{1}, m_{2}, \ldots, m_{n}\right) \in \mathbb{N}_{\geq 0}^{n}$ of $n$ non-negative integers, we define the (uncentered) moment of $\mathbf{x}$ associated with $\mathbf{m}$ to be

$$
\mu_{(\mathbf{m})}(t):=\mathbf{E}\left[\mathbf{x}^{(\mathbf{m})}(t)\right], \quad \forall t \geq 0
$$

where $\mathbf{E}$ stands for the expected value and $\mathbf{x}^{(\mathbf{m})}:=x_{1}^{m_{1}} x_{2}^{m_{2}} \cdots x_{n}^{m_{n}}$. The sum $\sum_{j=1}^{n} m_{j}$ is called the order of the moment. For example, consider a system of reactions with two species $(n=2)$ and $\mathbf{x}=\left[x_{1}, x_{2}\right]^{T}$. Then, the first order moments are given by

$$
\mu_{(1,0)}=\mathbf{E}\left[x_{1}\right], \quad \mu_{(0,1)}=\mathbf{E}\left[x_{2}\right]
$$

the second order moments are given by

$$
\mu_{(2,0)}=\mathbf{E}\left[x_{1}^{2}\right], \quad \mu_{(0,2)}=\mathbf{E}\left[x_{2}^{2}\right], \quad \mu_{(1,1)}=\mathbf{E}\left[x_{1} x_{2}\right],
$$


and so on. It is straightforward to derive the differential equation that describes the time evolution of the moment $\mathbf{E}\left[\mathbf{x}^{(\mathbf{m})}\right]$, which is given by

$$
\frac{d \mathbf{E}\left[\mathbf{x}^{(\mathbf{m})}\right]}{d t}=\mathbf{E}\left[\sum_{i=1}^{k} c_{i} h_{i}(\mathbf{x})\left\{\left(\mathbf{x}+\mathbf{a}_{i}\right)^{(\mathbf{m})}-\mathbf{x}^{(\mathbf{m})}\right\}\right]
$$

[9]. Assuming that there exists at least one reaction with a nonlinear propensity function $h_{i}(\mathbf{x})$, then it follows from (4) that the time derivative of the vector $\boldsymbol{\mu} \in \mathbb{R}^{k}$ consisting of the first $\mathscr{M}$ order moments of $\mathbf{x}$ is given by

$$
\dot{\boldsymbol{\mu}}=\hat{\mathbf{a}}+A \boldsymbol{\mu}+B \overline{\boldsymbol{\mu}}, \quad \boldsymbol{\mu}=\left[\boldsymbol{\mu}_{\left(\mathbf{m}_{1}\right)}, \ldots, \boldsymbol{\mu}_{\left(\mathbf{m}_{k}\right)}\right]^{T}, \quad \mathbf{m}_{p} \in \mathbb{N}_{\geq 0}^{n}, \quad \forall p \in\{1, \ldots, k\}
$$

for an appropriate constant vector $\hat{\mathbf{a}}$, constant matrices $A$ and $B$, and a (time-varying) vector $\bar{\mu} \in \mathbb{R}^{r}$ containing moments of order $\mathscr{M}+1$ [8]. One can see that the dynamics of vector $\boldsymbol{\mu}$ is not closed, in the sense that it depends on higher order moments in $\overline{\boldsymbol{\mu}}$. Our goal is to approximate (5) by a nonlinear system of the form

$$
\dot{\boldsymbol{v}}=\hat{\mathbf{a}}+A \boldsymbol{v}+B \overline{\boldsymbol{\varphi}}(\boldsymbol{v}), \boldsymbol{v}=\left[v_{\left(\mathbf{m}_{1}\right)}, \ldots, v_{\left(\mathbf{m}_{k}\right)}\right]^{T}
$$

where the map $\overline{\boldsymbol{\varphi}}: \mathbb{R}^{k} \rightarrow \mathbb{R}^{r}$ should be chosen so as to keep $\boldsymbol{v}(t)$ close to $\boldsymbol{\mu}(t)$. This procedure is commonly referred to as moment closure [6] and we refer to $\mathscr{M}$ as the order of the truncation.

To compute $\overline{\boldsymbol{\varphi}}$ we use the approach described in [9], where moment closure is done by matching time derivatives between $\boldsymbol{\mu}(t)$ and $\boldsymbol{v}(t)$ at some initial time $t_{0}$. In particular, assuming $\boldsymbol{\mu}\left(t_{0}\right)=\boldsymbol{v}\left(t_{0}\right)$, this derivative matching approach attempts to determine the map $\overline{\boldsymbol{\varphi}}$ for which

$$
\left.\frac{d^{i} \boldsymbol{\mu}(t)}{d t^{i}}\right|_{t=t_{0}}=\left.\frac{d^{i} \boldsymbol{v}(t)}{d t^{i}}\right|_{t=t_{0}}
$$

holds. The main rationale for doing so is that, if a sufficiently large number of derivatives of $\boldsymbol{\mu}(t)$ and $\boldsymbol{v}(t)$ match point-wise at an initial time $t_{0}$, then from a Taylor series argument the trajectories of $\boldsymbol{\mu}(t)$ and $\boldsymbol{v}(t)$ will remain close at least locally in time.

\section{SDM MOMENT CLOSURE PROCEDURE}

In this section we construct closed moment dynamics so that the condition (7) holds approximately. Let vector $\overline{\mathbf{m}} \in \mathbb{N}_{\geq 0}^{n}$ be such that, $\mu_{(\overline{\mathbf{m}})}$ is a moment of order $\mathscr{M}+1$ in $\overline{\boldsymbol{\mu}}$. The corresponding nonlinear 
approximation of $\mu_{(\overline{\mathbf{m}})}$ in the vector $\overline{\boldsymbol{\varphi}}(\boldsymbol{\mu})$, is denoted by $\varphi_{(\overline{\mathbf{m}})}(\boldsymbol{\mu})$. We refer to $\varphi_{(\overline{\mathbf{m}})}(\boldsymbol{\mu})$ as the moment closure function for the higher order moment $\mu_{(\overline{\mathbf{m}})}$. We seek for moment closure functions that have the following separable form

$$
\varphi_{(\overline{\mathbf{m}})}(\boldsymbol{\mu})=\prod_{p=1}^{k}\left(\mu_{\left(\mathbf{m}_{p}\right)}\right)^{\gamma_{p}}
$$

where $\gamma_{p}$ are appropriately chosen constants. In general, it will not be possible to find constants $\gamma_{p}$ such that (7) holds exactly for every initial condition. Thus we relax this condition and simply demand approximate derivative matching with deterministic initial conditions, i.e., $\mathbf{x}\left(t_{0}\right)=\mathbf{x}_{0}$ with probability one. In particular, we seek for constants $\gamma_{p}$ for which

$$
\boldsymbol{\mu}\left(t_{0}\right)=\left.\boldsymbol{v}\left(t_{0}\right) \Rightarrow \frac{d^{i} \boldsymbol{\mu}(t)}{d t^{i}}\right|_{t=t_{0}}=\left.\frac{d^{i} \boldsymbol{v}(t)}{d t^{i}}\right|_{t=t_{0}}+\boldsymbol{\varepsilon}_{i}\left(\mathbf{x}_{0}\right), \quad \forall i \in\{1,2, \ldots, N\}
$$

where each element of $\boldsymbol{\varepsilon}_{i}\left(\mathbf{x}_{0}\right)$ is a polynomial in $\mathbf{x}_{0}$. One can think of (9) as an approximation to (7) which will be valid as long as the moments in $\left.\frac{d^{i} \boldsymbol{\mu}(t)}{d t^{i}}\right|_{t=t_{0}}$ dominate over $\boldsymbol{\varepsilon}_{i}\left(\mathbf{x}_{0}\right)$.

Before stating our main result, for given vectors $\hat{\mathbf{m}}=\left(\hat{m}_{1}, \ldots, \hat{m}_{n}\right) \in \mathbb{N}_{\geq 0}^{n}$ and $\check{\mathbf{m}}=\left(\check{m}_{1}, \ldots, \check{m}_{n}\right) \in \mathbb{N}_{\geq 0}^{n}$, we define the scalar

$$
C_{(\grave{\mathbf{m}})}^{(\hat{\mathbf{m}})}:=C_{\check{m}_{1}}^{\hat{m}_{1}} C_{\check{m}_{2}}^{\hat{m}_{2}} \ldots C_{\check{m}_{n}}^{\hat{m}_{n}}
$$

where $C_{h}^{\ell}$ is defined as follows: $\forall \ell, h \in \mathbb{N}_{\geq 0}$

$$
C_{h}^{\ell}= \begin{cases}\frac{\ell !}{(\ell-h) ! h !}, & \ell \geq h \\ 0, & \ell<h\end{cases}
$$

and $\ell$ ! denotes the factorial of $\ell$. The factorial of zero is defined as one. Hence by this definition $C_{0}^{0}=1$.

Theorem 1: Assume that for each element $\mu_{(\overline{\mathbf{m}})}$ of $\overline{\boldsymbol{\mu}}$, the corresponding moment closure function $\varphi_{(\overline{\mathbf{m}})}$ in $\overline{\boldsymbol{\varphi}}$ is chosen according to (8) with $\gamma=\left(\gamma_{1}, \ldots, \gamma_{k}\right)$ taken as the unique solution of the following system of linear equations

$$
C_{\left(\mathbf{m}_{s}\right)}^{(\overline{\mathbf{m}})}=\sum_{p=1}^{k} \gamma_{p} C_{\left(\mathbf{m}_{s}\right)}^{\left(\mathbf{m}_{p}\right)}, \quad \forall s=\{1, \ldots, k\}
$$


Then, for every initial condition $\mathbf{x}\left(t_{0}\right)=\mathbf{x}_{0} \in \mathbb{R}^{n}$ with probability one, we have that

$$
\begin{aligned}
\boldsymbol{\mu}\left(t_{0}\right)=\boldsymbol{v}\left(t_{0}\right) & \left.\Rightarrow \frac{d \boldsymbol{\mu}(t)}{d t}\right|_{t=t_{0}}=\left.\frac{d \boldsymbol{v}(t)}{d t}\right|_{t=t_{0}} \\
& \left.\Rightarrow \frac{d^{2} \boldsymbol{\mu}(t)}{d t^{2}}\right|_{t=t_{0}}=\left.\frac{d^{2} \boldsymbol{v}(t)}{d t^{2}}\right|_{t=t_{0}}+\boldsymbol{\varepsilon}_{2}\left(\mathbf{x}_{0}\right)
\end{aligned}
$$

where all elements of $\boldsymbol{\varepsilon}_{2}\left(\mathbf{x}_{0}\right)$ are zero except the last $\bar{n}$ elements which are polynomials in $\mathbf{x}_{0}$ of degree 2. Here, $\bar{n}$ is the number of distinct moments of order $\mathscr{M}$.

The proof of this result is provided at the end of this Section. In the sequel we refer to moment closure functions obtained from (8) and (12) as Separable Derivative-Matching (SDM) moment closure functions. For example, for a second order truncation $(\mathscr{M}=2)$, the SDM moment closure functions that express various third order statistical moments of the population count in terms of the first and second order moments are as follows:

$$
\mathbf{E}\left[x_{1}^{3}\right] \approx\left(\frac{\mathbf{E}\left[x_{1}^{2}\right]}{\mathbf{E}\left[x_{1}\right]}\right)^{3}, \quad \mathbf{E}\left[x_{1}^{2} x_{2}\right] \approx\left(\frac{\mathbf{E}\left[x_{1}^{2}\right]}{\mathbf{E}\left[x_{2}\right]}\right)\left(\frac{\mathbf{E}\left[x_{1} x_{2}\right]}{\mathbf{E}\left[x_{1}\right]}\right)^{2}, \quad \mathbf{E}\left[x_{1} x_{2} x_{3}\right] \approx \frac{\mathbf{E}\left[x_{1} x_{2}\right] \mathbf{E}\left[x_{2} x_{3}\right] \mathbf{E}\left[x_{1} x_{3}\right]}{\mathbf{E}\left[x_{1}\right] \mathbf{E}\left[x_{2}\right] \mathbf{E}\left[x_{3}\right]}
$$

As the last $\bar{n}$ elements of $\boldsymbol{\mu}(t)$ contain moments of order $\mathscr{M}$, we have from (4) that the last $\bar{n}$ elements of the vector $\left.\frac{d^{2} \boldsymbol{\mu}(t)}{d t^{2}}\right|_{t=t_{0}}$ are polynomials in $\mathbf{x}_{0}$ of degree $\mathscr{M}+2$. Thus, for sufficiently large $\left\|\mathbf{x}_{0}\right\|$ we have that ${ }^{1}$

$$
\frac{\boldsymbol{\varepsilon}_{2}^{p}\left(\mathbf{x}_{0}\right)}{\left.\frac{d^{2} \mu_{\left(\mathbf{m}_{\mathbf{p}}\right)}(t)}{d t^{2}}\right|_{t=t_{0}}}=\left\{\begin{array}{ccc}
0 & \text { if } & p \leq k-\bar{n} \\
O\left(\left\|\mathbf{x}_{0}\right\|^{-\mathscr{M}}\right) & \text { if } & p \geq k-\bar{n}+1
\end{array}\right.
$$

where $\varepsilon_{2}^{p}\left(\mathbf{x}_{0}\right)$ and $\mu_{\left(\mathbf{m}_{\mathbf{p}}\right)}(t)$ denote the $p^{t h}$ element of the vector $\boldsymbol{\varepsilon}_{2}\left(\mathbf{x}_{0}\right)$ and $\boldsymbol{\mu}(t)$, respectively. This result shows that the error $\boldsymbol{\varepsilon}_{2}$ in the derivative-matching can be reduced by increasing the order of truncation $\mathscr{M}$.

It can be verified that with $\gamma_{p}$ chosen as in Theorem 1, the moment closure functions obtained also match all derivatives of order higher than 2 in (13b) with small errors. Using symbolic manipulation in Mathematica, for $n \in\{1,2,3\}$ and $i \in\{3,4,5\}$ one can verify that the degree of the polynomial elements of $\left.\frac{d^{i} \boldsymbol{\mu}(t)}{d t^{i}}\right|_{t=t_{0}}$ exceed by $\mathscr{M}$ the degree of the corresponding polynomial elements in the error vector $\boldsymbol{\varepsilon}_{i}\left(\mathbf{x}_{0}\right)=\left.\frac{d^{i} \boldsymbol{\mu}(t)}{d t^{i}}\right|_{t=t_{0}}-\left.\frac{d^{i} \boldsymbol{v}(t)}{d t^{i}}\right|_{t=t_{0}}$. Hence, by increasing $\mathscr{M}$, one can obtain truncated moment dynamics $\boldsymbol{v}(t)$ that provide more accurate approximations to the lower order moments $\boldsymbol{\mu}(t)$. We conjecture that this

\footnotetext{
${ }^{1}$ We recall the "big-O" notation, according to which $f(z)=O(g(z))$ means that $\exists z_{0}, C>0$ such that $|f(z)| \leq C|g(z)|, \forall z \geq z_{0}$.
} 
is true $\forall n \in \mathbb{N}$ and $\forall i \in \mathbb{N}$ but we only verified it for $n$ up to 3 and $i$ up to 5 .

One can also verify that the dependence of higher-order moments on lower order ones is consistent with log-normal distributions, i.e., $\mu_{(\overline{\mathbf{m}})}=\varphi_{\overline{\mathbf{m}}}(\boldsymbol{\mu})$ is an exact equation for log-normal distributions. An important implication of this is that if the population distribution was log-normal at all times, then our moment closure procedure would be exact. However, in most cases it is straightforward to verify that the distributions are not log-normal. In this case, this moment closure method only provides approximate moment dynamics, and the accuracy of the approximation can be arbitrarily improved by increasing the order of truncation. It is important to emphasize that the fact that the moment closure functions are consistent with log-normal distributions does not mean that the approximate moments $\boldsymbol{v}(t)$ are consistent with the population being log-normally distributed. It is precisely because of this that as we increase the order of truncation the closed moment dynamics $\boldsymbol{v}(t)$ provides more accurate estimates of the actual moment dynamics by deviating further from a log-normal distribution.

The procedure described here to generate approximated moment dynamics can be fully automated. The software [12] is available to compute closed moment dynamics for any order of truncation starting from a simple ASCII description of the chemical reactions involved.

Proof of Theorem 1: We will use the notation $\mathbf{m}_{p} \in \mathscr{O}_{m}$ to denote the fact that $\mu_{\left(\mathbf{m}_{p}\right)}$ is a moment of order $m$ and we shall assume that the moments in vector $\boldsymbol{\mu}$ are arranged in increasing order, i.e., $\mathbf{m}_{p} \in \mathscr{O}_{1}, \quad \forall p \in\{1, \ldots, n\}, \mathbf{m}_{p} \in \mathscr{O}_{2}, \quad \forall p \in\{n+1, \ldots, n+n(n+1) / 2\}$ and so on. Moreover, the vector $\mathbf{m}_{p} \in \mathscr{O}_{1}$ has 1 in the $p^{\text {th }}$ position and all other entries are zero. From (5) and (6) it is sufficient to prove the following : For each element $\mu_{(\overline{\mathbf{m}})}$ of $\overline{\boldsymbol{\mu}}$ and its corresponding moment closure function $\varphi_{(\overline{\mathbf{m}})}(\boldsymbol{\mu})$ we have

$$
\begin{aligned}
& \mu_{(\overline{\mathbf{m}})}\left(t_{0}\right)=\varphi_{(\overline{\mathbf{m}})}\left(\boldsymbol{\mu}\left(t_{0}\right)\right), \\
& \left.\frac{d \mu_{(\overline{\mathbf{m}})}(t)}{d t}\right|_{t=t_{0}}=\left.\frac{d \varphi_{(\overline{\mathbf{m}})}(\boldsymbol{\mu}(t))}{d t}\right|_{t=t_{0}}+\bar{\varepsilon}\left(\mathbf{x}_{0}\right)
\end{aligned}
$$

where the scalar $\bar{\varepsilon}\left(\mathbf{x}_{0}\right)$ is polynomial in $\mathbf{x}_{0}$ of degree 2 . We first prove (16a). For the initial conditions $\mathbf{x}\left(t_{0}\right)=\mathbf{x}_{0}$ with probability one and using (8) we have

$$
\mu_{(\overline{\mathbf{m}})}\left(t_{0}\right)=\mathbf{x}_{0}^{(\overline{\mathbf{m}})}, \quad \varphi_{(\overline{\mathbf{m}})}\left(\boldsymbol{\mu}\left(t_{0}\right)\right)=\prod_{p=1}^{k}\left(\mathbf{x}_{0}^{\left(\mathbf{m}_{p}\right)}\right)^{\gamma_{p}}=\mathbf{x}_{0}^{\left(\sum_{p=1}^{k} \gamma_{p} \mathbf{m}_{p}\right)}
$$


Using (10) and the fact that the vectors $\mathbf{m}_{p} \in \mathscr{O}_{1}, p \in\{1, \ldots, n\}$ have 1 in the $p^{t h}$ position and all other entries zero, we have that

$$
\overline{\mathbf{m}}=\left(C_{\left(\mathbf{m}_{1}\right)}^{(\overline{\mathbf{m}})}, \ldots, C_{\left(\mathbf{m}_{n}\right)}^{(\overline{\mathbf{m}})}\right), \quad \mathbf{m}_{p}=\left(C_{\left(\mathbf{m}_{1}\right)}^{\left(\mathbf{m}_{p}\right)}, \ldots, C_{\left(\mathbf{m}_{n}\right)}^{\left(\mathbf{m}_{p}\right)}\right), \quad \forall p \in\{1, \ldots, k\}
$$

From the above equalities and using (12) for $s \in\{1, \ldots, n\}$ we have that $\overline{\mathbf{m}}=\sum_{p=1}^{k} \gamma_{p} \mathbf{m}_{p}$. Hence from (17) equality (16a) holds. Our next goal is to prove (16b). A binomial expansion of (4) yields the following time derivative of a moment $\mu_{(\mathbf{m})}$ of order $m$ :

$$
\frac{d \mu_{(\mathbf{m})}(t)}{d t}=\mathbf{E}\left[\sum_{i=1}^{K} c_{i} h_{i}(\mathbf{x}) g_{i}(\mathbf{x})\right], \quad g_{i}(\mathbf{x})=\sum_{s=1}^{k} C_{\left(\mathbf{m}_{s}\right)}^{(\mathbf{m})} \mathbf{x}^{\left(\mathbf{m}-\mathbf{m}_{s}\right)} \mathbf{a}_{i}^{\left(\mathbf{m}_{s}\right)}+P_{m-1-\mathscr{M}}(\mathbf{x})
$$

where $P_{m-1-\mathscr{M}}(\mathbf{x})$ is zero if $m-1-\mathscr{M}<0$, constant if $m-1-\mathscr{M}=0$ and a polynomial in $\mathbf{x}$ of degree $m-1-\mathscr{M}$ otherwise. As $\mu_{(\overline{\mathbf{m}})}$ is a moment of order $\mathscr{M}+1$ ( hence $m=\mathscr{M}+1$ ) we have from (19)

$$
\left.\frac{d \mu_{(\overline{\mathbf{m}})}(t)}{d t}\right|_{t=t_{0}}=\sum_{i=1}^{K} c_{i} h_{i}\left(\mathbf{x}_{0}\right)\left\{\sum_{s=1}^{k} C_{\left(\mathbf{m}_{s}\right)}^{(\overline{\mathbf{m}})} \mathbf{x}_{0}^{\left(\overline{\mathbf{m}}-\mathbf{m}_{s}\right)} \mathbf{a}_{i}^{\left(\mathbf{m}_{s}\right)}+\beta_{i}\right\}
$$

where $\beta_{i}$ is a constant. Also from (8) and using (16a), (19)

$$
\begin{aligned}
\left.\frac{d \varphi_{(\overline{\mathbf{m}})}(\boldsymbol{\mu}(t))}{d t}\right|_{t=t_{0}} & =\left.\varphi_{(\overline{\mathbf{m}})}\left(\boldsymbol{\mu}\left(t_{0}\right)\right) \sum_{p=1}^{k} \gamma_{p} \frac{d \mu_{\left(\mathbf{m}_{p}\right)}\left(t_{0}\right)}{d t}\right|_{t=t_{0}} / \mu_{\left(\mathbf{m}_{p}\right)}\left(t_{0}\right)=\left.\sum_{p=1}^{k} \gamma_{p} \mathbf{x}_{0}^{\left(\overline{\mathbf{m}}-\mathbf{m}_{p}\right)} \frac{d \mu_{\left(\mathbf{m}_{p}\right)}(t)}{d t}\right|_{t=t_{0}} \\
& =\sum_{i=1}^{K} c_{i} h_{i}\left(\mathbf{x}_{0}\right)\left\{\sum_{s=1}^{k} \sum_{p=1}^{k} \gamma_{p} C_{\left(\mathbf{m}_{s}\right)}^{\left(\mathbf{m}_{p}\right)} \mathbf{x}_{0}^{\left(\overline{\mathbf{m}}-\mathbf{m}_{s}\right)} \mathbf{a}_{i}^{\left(\mathbf{m}_{s}\right)}\right\}=\sum_{i=1}^{K} c_{i} h_{i}\left(\mathbf{x}_{0}\right)\left\{\sum_{s=1}^{k} C_{\left(\mathbf{m}_{s}\right)}^{\left(\overline{\mathbf{m}}_{p}\right)} \mathbf{x}_{0}^{\left(\overline{\mathbf{m}}-\mathbf{m}_{s}\right)} \mathbf{a}_{i}^{\left(\mathbf{m}_{s}\right)}\right\}
\end{aligned}
$$

Using (12), (20) and (21) one can see that

$$
\bar{\varepsilon}\left(\mathbf{x}_{0}\right)=\sum_{i=1}^{K} c_{i} h_{i}\left(\mathbf{x}_{0}\right) \beta_{i}
$$

and is a polynomial of degree 2 .

\section{EXAmple}

In this section we consider the following set of chemical reactions

$$
* \stackrel{c_{1}}{\longrightarrow} B_{1} X_{1}, \quad 2 X_{1} \stackrel{c_{2}}{\longrightarrow}, 2 X_{1} \stackrel{c_{3}}{\longrightarrow} 2 X_{1}+B_{2} X_{2}, \quad X_{2} \stackrel{c_{4}}{\longrightarrow} .
$$


This example is motivated by a gene cascade network where a gene (denoted by the symbol $*$ ) expresses a protein $X_{1}$ at a rate $c_{1}$ and each expression event produces $B_{1}$ molecules of the protein. The protein $X_{1}$ undergoes dimerization to form a functionally active dimer which decays at a constant rate $c_{2}$. We assume that the protein can only be used in its dimer form and the kinetics of protein dimerization is sufficiently fast. These assumptions result in the second reaction in (23). The protein $X_{1}$ dimer also activates another gene to express the protein $X_{2}$ with each expression event producing $B_{2}$ molecules of $X_{2}$. The parameters given by $c_{1}=50 \mathrm{~V}, c_{2}=0.8 / \mathrm{V}, c_{3}=0.04 / \mathrm{V}, c_{4}=15$ are defined in terms of the volume $V$. This particular scaling of the rate parameters with $V$, will essentially keep the average concentrations constant (i.e., number of molecules per unit of volume), and therefore a small volume corresponds to small steady-state populations.

We consider two different moment closure methods: the SDM moment closure method developed in this paper and another method where moment closure functions are constructed by setting the statistical cumulants of the population equal to zero [10], [11], [13]. This latter method is referred to as the zerocumulant moment closure and here a $\mathscr{M}^{\text {th }}$ order truncation is performed by setting the $\mathscr{M}+1^{\text {th }}$ order cumulant of $\mathbf{x}$ equal to zero. Since for a Gaussian distribution all cumulants of order three and higher are equal to zero, the zero-cumulant moment closure is consistent with the population count $\mathbf{x}$ being jointly gaussian.
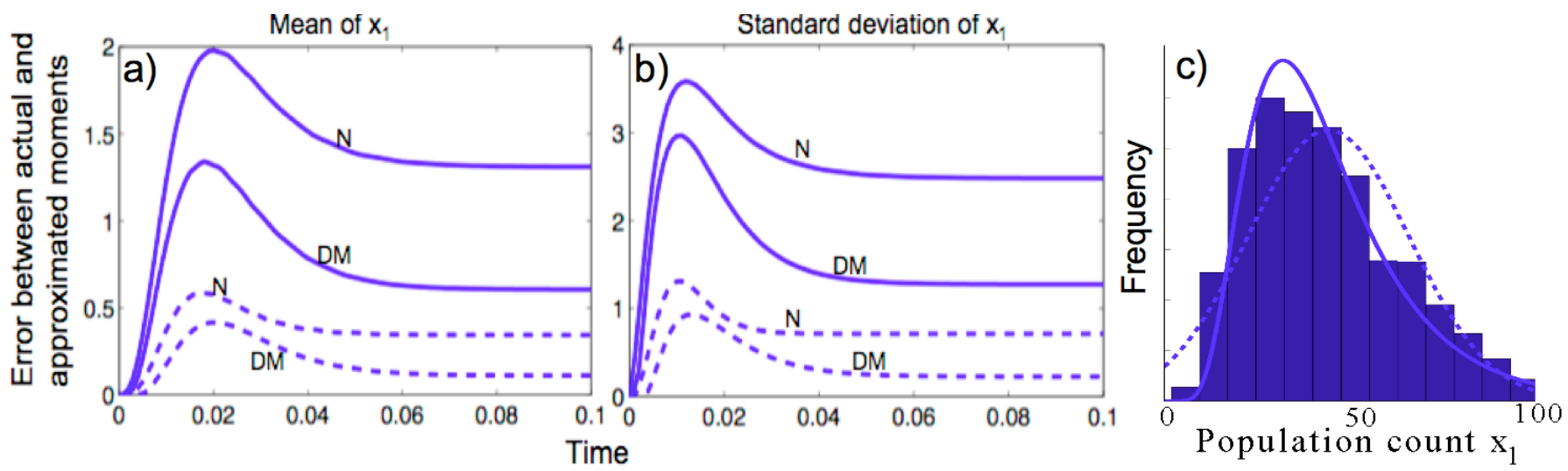

Fig. 1. Plots of the absolute value of the error between the actual and approximated moment dynamics for the mean of $x_{1}$ (a) and the standard deviation of $x_{1}$ (b) corresponding to a second (solid lines) and a third order (dashed lines) truncation. DM and $\mathrm{N}$ refer to the SDM and zero-cumulant moment closure, respectively. Initial conditions were taken as follows: $x_{1}(0)$ is binomially distributed with a mean of 10 and variance of $5 ; x_{2}(0)$ is binomially distributed with a mean of 25 and variance of 12.5 . The actual moment estimates were obtained from the SDM moment closure using a fifth order truncation. The burst sizes $B_{1}$ and $B_{2}$ were taken as 40 and 15 , respectively. c) Steady-states histogram for $x_{1}$, the number of molecules of protein $X_{1}$. The solid and dashed line corresponds to a lognormal and normal distribution approximation of the histogram, respectively, with mean and standard deviations obtained from the corresponding closed moment dynamics.

We first take $V=1$ units, which corresponds to a small average steady-state populations of about 50 


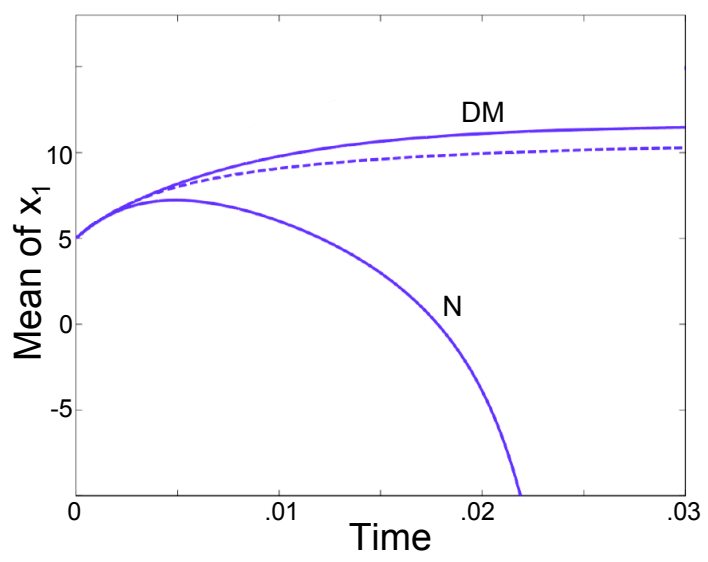

Fig. 2. Time evolution of the mean of $x_{1}$ obtained from a second order SDM moment closure (DM), second order zero-cumulant moment closure $(\mathrm{N})$ when the population size is very small $(V=0.2$ units). The dashed line corresponds to the actual time evolution of the mean of $x_{1}$ obtained from the SDM moment closure using a fifth order truncation. The initial conditions were taken as $x_{1}(0)=x_{2}(0)=5$ molecules.

molecules, for both species. Figure 1 plots the moment estimates for $x_{1}$ corresponding to a second order SDM and zero-cumulant moment closure method $(\mathscr{M}=2)$. Note that in this small-population regime the SDM moment closure yields smaller errors between the approximated and actual moment dynamics compared to the zero-cumulant moment closure. The explanation for this can be deduced from Figure 1c, which plots the steady-state distribution of $x_{1}$ obtained from a large number of Monte Carlo simulations (Gillespie's SSA [1]). This positively skewed distribution closely approximates a lognormal distribution, and hence, the SDM moment closure functions which are consistent with $\mathbf{x}$ being jointly lognormally distributed provide better moment estimates. Notice from Figure 1c that the corresponding normal distribution approximation (dashed line) has some portion in the negative region, which is not biologically meaningful since molecule populations cannot drop below zero. Unlike the lognormal distribution which is only defined for positive values, this is frequently a problem with the normal approximation when the average number of molecules is small.

We next increase the order of truncation by performing a third order truncation $(\mathscr{M}=3)$. Figure 1 confirms that increasing the order of truncation reduces the errors and considerably improves the moment estimates from the closed moment dynamics.

Recent results have pointed out that for small population sizes, the zero-cumulant moment closure can be dynamically unstable and provide unbounded solutions that are not physically meaningful [6]. We investigate this small-population instability by taking $V=0.2$ units which corresponds to an average steady-state of about 10 molecules for both species. Consistent with observations in [6], moment estimates from the zero-cumulant moment closure blow up to negative infinity (see Figure 2). On the other hand, 
moment estimates from the SDM moment closure remain bounded and provided a good estimate of the actual moments. In summary, the results of this section have shown that for small population sizes, the SDM moment closure has better performance, both in terms of more accurate moment estimation and higher stability of the closed moment dynamics, compared to the zero-cumulant moment closure.

\section{CONCLUSiOn AND Future WORK}

A procedure for estimating the statistical moments for the number of molecules of different species involved in a chemical reaction was presented. This was done by first obtaining the differential equations that describe the time evolution of the moments, and then closing them using moment closure. The main result of this paper is Theorem 1 which provides explicit formulae to compute the SDM moment closure functions that approximate higher order moments in $\bar{\mu}$ as nonlinear functions of the lower order moments in $\boldsymbol{\mu}$, for any order of truncation $\mathscr{M}$. An important feature of the proposed moment closure method is that its accuracy can be considerably improved by increasing the order of truncation $\mathscr{M}$. However, this comes at the cost of obtaining higher-dimensional systems as the size of the closed moment dynamics also increases with $\mathscr{M}$.

SDM moment closure functions were shown to be consistent with lognormal distributions. This property makes the SDM moment closure produce especially well suited for reactions with small population sizes that result in positively skewed population distributions. Moment closure based on lognormal distributions has been previously reported in literature. In particular, [7] derived moment closure functions corresponding to a second order truncation $(\mathscr{M}=2)$ by directly assuming that the population distribution is lognormal. This assumption by itself cannot be used to determine unique moment closure functions for higher order truncations $(\mathscr{M} \geq 3)$. This is because for a lognormal distribution, the $\mathscr{M}+1^{\text {th }}$ order moments (where $\mathscr{M} \geq 3$ ) can be expressed in infinitely many ways as a function of the first $\mathscr{M}$ order moments. The procedure used in this paper is necessary to derive unique moment closure functions consistent with the lognormal distribution that perform derivative matching, and hence provide accurate moment estimates at least locally in time. Our work thus constitutes an important extension of previous work by deriving unique moment closure formulas for any arbitrary order of truncation and any number of species.

Reactions where species can go extinct (or population count goes to zero) with high probability pose a limitation to our moment closure scheme. In such cases, the truncated moment dynamics can either become unstable or a very large order of truncation is needed to capture the moment dynamics. It turns 
out that most closure schemes fail to capture such stochastic extinctions, and those available are restricted to single specie reactions only [14]. Alternative moment closure functions based on derivative matching are currently being explored for such reactions.

\section{ACKNOWLEDGMENT}

J.P.H acknowledges funding from the Institute for Collaborative Biotechnologies through grant DAAD19-03-D-0004 from the U.S. Army Research Office and by the National Science Foundation under Grant numbers ECCS-0725485 and ECCS-0835847.

\section{REFERENCES}

[1] D. T. Gillespie, "A general method for numerically simulating the stochastic time evolution of coupled chemical reactions," J. of Computational Physics, vol. 22, pp. 403-434, 1976.

[2] M. A. Gibson and J. Bruck, "Efficient exact stochastic simulation of chemical systems with many species and many channels," J. of Physical Chemistry A, vol. 104, pp. 1876-1889, 2000.

[3] Y. Cao, H. Li, and L. Petzold, "Efficient formulation of the stochastic simulation algorithm for chemically reacting systems," J. of Chemical Physics, vol. 121, pp. 4059-4067, 2004.

[4] B. Munsky and M. Khammash, "The finite state projection algorithm for the solution of the chemical master equation," J. of Chemical Physics, vol. 124, 2006.

[5] N. G. V. Kampen, Stochastic Processes in Physics and Chemistry. Amsterdam, The Netherlands: Elsevier Science, 2001.

[6] I. Nasell, "An extension of the moment closure method," Theoretical Population Biology, vol. 64, pp. $233-239,2003$.

[7] M. J. Keeling, "Multiplicative moments and measures of persistence in ecology," J. of Theoretical Biology, vol. 205, pp. 269-281, 2000.

[8] A. Singh and J. P. Hespanha, "Lognormal moment closures for biochemical reactions." in Proc. of the 45th Conf. on Decision and Control, San Diego, 2006.

[9] J. P. Hespanha and A. Singh, "Stochastic models for chemically reacting systems using polynomial stochastic hybrid systems," Int. J. of Robust and Nonlinear Control, vol. 15, pp. 669-689, 2005.

[10] C. A. Gomez-Uribe and G. C. Verghese, "Mass fluctuation kinetics: Capturing stochastic effects in systems of chemical reactions through coupled mean-variance computations," J. of Chemical Physics, vol. 126, 2007.

[11] C. H. Lee, K. Kim, and P. Kim, “A moment closure method for stochastic reaction networks," J. of Chemical Physics, vol. 130, p. 134107, 2009.

[12] J. P. Hespanha, "StochDynTools - a MATLAB toolbox to compute moment dynamics for stochastic networks of bio-chemical reactions," 2006, available at http://www.ece.ucsb.edu/ hespanha/software/stochdyntool.html.

[13] J. Goutsias, "Classical versus stochastic kinetics modeling of biochemical reaction systems," Biophysical Journal, vol. 92, pp. 2350$2365,2007$.

[14] I. Krishnarajah, A. Cook, G. Marion, and G. Gibson, "Novel moment closure approximations in stochastic epidemics," Bulletin of Mathematical Biology, vol. 67, pp. 855-873, 2005. 